\title{
SosEmanuk, a fast software-oriented stream cipher $^{\star}$
}

\author{
C. Berbain ${ }^{1}$, O. Billet ${ }^{1}$, A. Canteaut ${ }^{2}$, N. Courtois $^{3}$, H. Gilbert ${ }^{1}$, L. \\ Goubin $^{4}$, A. Gouget ${ }^{5}$, L. Granboulan ${ }^{6}$, C. Lauradoux ${ }^{2}$, M. Minier ${ }^{7}$, T. \\ Pornin $^{8}$, and H. Sibert ${ }^{9}$ \\ 1 Orange Labs, France, \\ \{come.berbain, olivier.billet, henri.gilbert\}@orange-ftgroup.com \\ ${ }^{2}$ INRIA-Rocquencourt, projet CODES, France, \{anne.canteaut, \\ cedric.lauradoux\}@inria.fr \\ 3 University College of London, UK, n.courtois@ucl.ac.uk \\ ${ }^{4}$ Université de Versailles, France, louis.goubin@prism.uvsq.fr \\ ${ }^{5}$ Gemalto, France, aline.gouget@gemalto.com \\ 6 EADS, France, louis.granboulan@eads.net \\ 7 INSA de Lyon, France, marine.minier@insa-lyon.fr \\ 8 Cryptolog International, France, thomas.pornin@cryptolog.com \\ ${ }_{9}$ NXP Semiconductors, France, herve.sibert@nxp.com
}

\begin{abstract}
Sosemanuk is a new synchronous software-oriented stream cipher, corresponding to Profile 1 of the ECRYPT call for stream cipher primitives. Its key length is variable between 128 and 256 bits. It accommodates a 128-bit initial value. Any key length is claimed to achieve 128-bit security. The Sosemanuk cipher uses both some basic design principles from the stream cipher SNOW 2.0 and some transformations derived from the block cipher SERPENT. SosEMANUK aims at improving SNOW 2.0 both from the security and from the efficiency points of view. Most notably, it uses a faster IV-setup procedure. It also requires a reduced amount of static data, yielding better performance on several architectures.
\end{abstract}

\section{Introduction}

This paper presents a proposal for a new synchronous software-oriented stream cipher, named Sosemanuk. The Sosemanuk cipher uses both basic design principles from the stream cipher SNOW 2.0 [12] and transformations derived from the block cipher SERPENT [3]. For this reason, its name should refer both to SERPENT and SNOW. However, it is wellknown that snow snakes do not exist since snakes either hibernate or

\footnotetext{
* Work partially supported by the French Ministry of Research RNRT Project "XCRYPT" and by the European Commission via ECRYPT network of excellence IST-2002-507932.
} 
move to warmer climes during the winter.Instead SosemanuK is a popular sport played by the Eastern Canadian tribes. It consists in throwing a wooden stick along a snow bank as far as possible. Its name means snowsnake in the Cree language, since the stick looks like a snake in the snow. Kwakweco-cime win is a variant of the same game but does not sound like an appropriate cipher name. More details on the SosEmanuK game and a demonstration can be found in [19] and [24].

The Sosemanuk stream cipher is a new synchronous stream cipher dedicated to software applications. Its key length is variable between 128 and 256 bits. Any key length is claimed to achieve 128-bit security. It is inspired by the design of SNOW 2.0 which is very elegant and achieves a very high throughput on a Pentium 4. SosEmanuk aims at improving SNOW 2.0 from two respects. First, it avoids some structural properties which may appear as potential weaknesses, even if the SNOW 2.0 cipher with a 128-bit key resists all known attacks. Second, efficiency is improved on several architectures by reducing the internal state size, thus allowing for a more direct mapping of data on the processor registers. SosEMANUK also requires a reduced amount of static data; this lower data cache pressure yields better performance on several architectures. Another strength of Sosemanuk is that its key setup procedure is based on a reduced version of the well-known block cipher SERPENT, improving classical initialization procedures both from an efficiency and a security point of view.

\section{Specification}

\subsection{SERPENT and derivatives}

SERPENT [3] is a block cipher proposed as an AES candidate. SERPENT operates over blocks of 128 bits which are split into four 32-bit words, which are then combined in so-called "bitslice" mode. SERPENT can thus be defined as working over quartets of 32-bit words. We number SERPENT input and output quartets from 0 to 3 , and write them in the order: $\left(Y_{3}, Y_{2}, Y_{1}, Y_{0}\right) . Y_{0}$ is the least significant word, and contains the least significant bits of the 32 4-bit inputs to the SERPENT S-boxes. When SERPENT output is written into 16 bytes, the $Y_{i}$ values are written following the little-endian convention (least significant byte first), and $Y_{0}$ is output first, then $Y_{1}$, and so on.

From SERPENT, we define two primitives called Serpent1 and Serpent24. 
Serpent1 A SERPENT rounds consist of, in that order:

- a subkey addition, by bitwise exclusive or;

- S-box application (which is expressed as a set of bitwise combinations between the four running 32-bit words, in bitslice mode);

- a linear bijective transformation (which amounts to a few XORs, shifts and rotations in bitslice mode), see Appendix A.2.

Serpent1 is one round of SERPENT, without the key addition and the linear transformation. SERPENT uses eight distinct S-boxes (see A.1 for details), numbered from $S_{0}$ to $S_{7}$ on 4-bit words. We define Serpent1 as the application of $S_{2}$, in bitslice mode. This is the third S-box layer of SERPENT. Serpent1 takes four 32-bit words as input, and provides four 32-bit words as output.

Serpent24 Serpent24 is SERPENT reduced to 24 rounds, instead of the 32 rounds of the full version of SERPENT. Serpent24 is equal to the first 24 rounds of SERPENT, where the last round (the 24th) is a complete one and includes a complete round with the linear transformation and an XOR with the 25th subkey. In other words, the 24th round of Serpent24 is thus equivalent to the thirty-second round of SERPENT, except that it contains the linear transformation and that the 24th and 25th subkeys are used (32nd and 33rd subkeys in SERPENT). Thus, the last round equation on Page 224 in [3] is

$$
R_{23}(X)=L\left(\hat{S}_{23}\left(X \oplus \hat{K}_{23}\right)\right) \oplus \hat{K}_{24} .
$$

Serpent24 uses only 25 128-bit subkeys, which are the first 25 subkeys produced by the SERPENT key schedule. In Sosemanuk, Serpent24 is used for the initialization step, only in encryption mode. Decryption is not used.

\subsection{The LFSR}

Underlying finite field Most of the stream cipher internal state is held in a LFSR containing 10 elements of $\mathbb{F}_{2^{32}}$, the field with $2^{32}$ elements. The elements of $\mathbb{F}_{2^{32}}$ are represented exactly as in SNOW 2.0. We recall this representation here. Let $\mathbb{F}_{2}$ denote the finite field with 2 elements. Let $\beta$ be a root of the primitive polynomial:

$$
Q(X)=X^{8}+X^{7}+X^{5}+X^{3}+1
$$


on $\mathbb{F}_{2}[X]$. We define the field $\mathbb{F}_{2^{8}}$ as the quotient $\mathbb{F}_{2}[X] / Q(X)$. Each element in $\mathbb{F}_{2^{8}}$ is represented using the basis $\left(\beta^{7}, \beta^{6}, \ldots \beta, 1\right)$. Since the chosen polynomial is primitive, then $\beta$ is a multiplicative generator of all invertible elements of $\mathbb{F}_{2^{8}}$ : every non-zero element in $\mathbb{F}_{2^{8}}$ is equal to $\beta^{k}$ for some integer $k(0 \leq k \leq 254)$. Any element in $\mathbb{F}_{2^{8}}$ is identified with an 8-bit integer by the following bijection:

$$
\begin{aligned}
\phi: \mathbb{F}_{2^{8}} & \rightarrow\{0,1, \ldots, 255\} \\
x=\sum_{i=0}^{7} x_{i} \beta^{i} & \mapsto \sum_{i=0}^{7} x_{i} 2^{i}
\end{aligned}
$$

where each $x_{i}$ is either 0 or 1 . For instance, $\beta^{23}$ is represented by the integer $\phi\left(\beta^{23}\right)=0 \times 1$ (in hexadecimal). Therefore, the addition of two elements in $\mathbb{F}_{2^{8}}$ corresponds to a bitwise XOR between the corresponding integer representations. The multiplication by $\beta$ is a left shift by one bit of the integer representation, followed by an XOR with a fixed mask if the most significant bit dropped by the shift equals 1 .

Let $\alpha$ be a root of the primitive polynomial

$$
P(X)=X^{4}+\beta^{23} X^{3}+\beta^{245} X^{2}+\beta^{48} X+\beta^{239}
$$

on $\mathbb{F}_{2^{8}}[X]$. The field $\mathbb{F}_{2^{32}}$ is then defined as the quotient $\mathbb{F}_{2^{8}}[X] / P(X)$, i.e., its elements are represented with the basis $\left(\alpha^{3}, \alpha^{2}, \alpha, 1\right)$. Any element in $\mathbb{F}_{2^{32}}$ is identified with a 32-bit integer by the following bijection:

$$
\begin{aligned}
\psi: \mathbb{F}_{2^{32}} & \rightarrow\left\{0,1, \ldots, 2^{32}-1\right\} \\
y=\sum_{i=0}^{3} y_{i} \alpha^{i} & \mapsto \sum_{i=0}^{3} \phi\left(y_{i}\right) 2^{8 i}
\end{aligned}
$$

Thus, the addition of two elements in $\mathbb{F}_{2^{32}}$ corresponds to a bitwise XOR between their integer representations. This operation will hereafter be denoted by $\oplus$. SosEmanuk also uses multiplications and divisions of elements in $\mathbb{F}_{2^{32}}$ by $\alpha$. Multiplication of $z \in \mathbb{F}_{2^{32}}$ by $\alpha$ corresponds to a left shift by 8 bits of $\psi(z)$, followed by an XOR with a 32-bit mask which depends only on the most significant byte of $\psi(z)$. Division of $z \in \mathbb{F}_{2^{32}}$ by $\alpha$ is a right shift by 8 bits of $\psi(z)$, followed by an XOR with a 32-bit mask which depends only on the least significant byte of $\psi(z)$.

Definition of the LFSR The LFSR operates over elements of $\mathbb{F}_{2^{32}}$. The initial state, at $t=0$, entails the ten 32 -bit values $s_{1}$ to $s_{10}$. At each step, a new value is computed, with the following recurrence:

$$
s_{t+10}=s_{t+9} \oplus \alpha^{-1} s_{t+3} \oplus \alpha s_{t}, \quad \forall t \geq 1
$$

and the register is shifted (see Figure 1 for an illustration of the LFSR). 


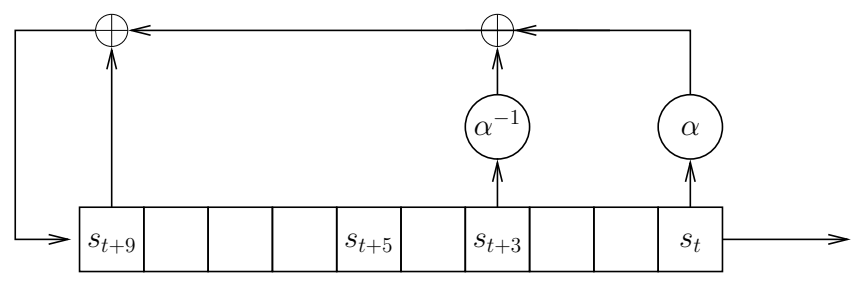

Fig. 1. The LFSR

The LFSR is associated with the following feedback polynomial:

$$
\pi(X)=\alpha X^{10}+\alpha^{-1} X^{7}+X+1 \in \mathbb{F}_{2^{32}}[X]
$$

Since the LFSR is non-singular and since $\pi$ is a primitive polynomial, the sequence of 32 -bit words $\left(s_{t}\right)_{t \geq 1}$ is periodic and has maximal period $\left(2^{320}-1\right)$.

\subsection{The Finite State Machine}

The Finite State Machine (FSM) is a component with 64 bits of memory, corresponding to two 32-bit registers $R 1$ and $R 2$. At each step, the FSM takes as inputs some words from the LFSR state; it updates the memory bits and produces a 32-bit output. The FSM operates on the LFSR state at time $t \geq 1$ as follows:

$$
F S M_{t}:\left(R 1_{t-1}, R 2_{t-1}, s_{t+1}, s_{t+8}, s_{t+9}\right) \mapsto\left(R 1_{t}, R 2_{t}, f_{t}\right)
$$

where

$$
\begin{aligned}
R 1_{t} & =\left(R 2_{t-1}+\operatorname{mux}\left(\operatorname{lsb}\left(R 1_{t-1}\right), s_{t+1}, s_{t+1} \oplus s_{t+8}\right)\right) \bmod 2^{32} \\
R 2_{t} & =\operatorname{Trans}\left(R 1_{t-1}\right) \\
f_{t} & =\left(s_{t+9}+R 1_{t} \bmod 2^{32}\right) \oplus R 2_{t}
\end{aligned}
$$

where $\operatorname{lsb}(x)$ is the least significant bit of $x, \operatorname{mux}(c, x, y)$ is equal to $x$ if $c=0$, or to $y$ if $c=1$. The internal transition function Trans on $\mathbb{F}_{2^{32}}$ is defined by

$$
\operatorname{Trans}(z)=\left(M \times z \bmod 2^{32}\right)_{<<<7}
$$

where $M$ is the constant value $0 \times 54655307$ (the hexadecimal expression of the first ten decimals of $\pi$ ) and $<<<$ denotes bitwise rotation of a 32-bit value (by 7 bits here). 


\subsection{Output transformation}

The outputs of the FSM are grouped by four, and Serpent1 is applied to each group; the result is then combined by XOR with the corresponding dropped values from the LFSR, to produce the output values $z_{t}$ :

$$
\left(z_{t+3}, z_{t+2}, z_{t+1}, z_{t}\right)=\operatorname{Serpent1}\left(f_{t+3}, f_{t+2}, f_{t+1}, f_{t}\right) \oplus\left(s_{t+3}, s_{t+2}, s_{t+1}, s_{t}\right)
$$

Four consecutive rounds of SosEmANuk are depicted in Figure 2 .

\subsection{SosemanuK workflow}

The Sosemanuk cipher combines the FSM and the LFSR to produce the output values $z_{t}$. Time $t=0$ designates the internal state after initialization; the first output value is $z_{1}$. Figure 目 gives a graphical overview of SOSEMANUK.

At time $t \geq 1$, we perform the following operations:

- The FSM is updated: $R 1_{t}, R 2_{t}$ and the intermediate value $f_{t}$ are computed from $R 1_{t-1}, R 2_{t-1}, s_{t+1}, s_{t+8}$ and $s_{t+9}$.

- The LFSR is updated: $s_{t+10}$ is computed, from $s_{t}, s_{t+3}$ and $s_{t+9}$. The value $s_{t}$ is sent to an internal buffer, and the LFSR is shifted.

Once every four steps, four output values $z_{t}, z_{t+1}, z_{t+2}$ and $z_{t+3}$ are produced from the accumulated values $f_{t}, f_{t+1}, f_{t+2}, f_{t+3}$ and $s_{t}, s_{t+1}, s_{t+2}, s_{t+3}$. Thus, Sosemanuk produces 32-bit values. We recommend encoding them into groups of four bytes using the little-endian convention, because it is faster on the most widely used high-end software platform (x86-compatible PC), and because SERPENT uses that convention.

Therefore, the first four iterations of SosEMANUK are as follows.

- The LFSR initial state contains values $s_{1}$ to $s_{10}$; no value $s_{0}$ is defined. The FSM initial state contains $R 1_{0}$ and $R 2_{0}$.

- During the first step, $R 1_{1}, R 2_{1}$ and $f_{1}$ are computed from $R 1_{0}, R 2_{0}$, $s_{2}, s_{9}$ and $s_{10}$.

- The first step produces the buffered intermediate values $s_{1}$ and $f_{1}$.

- During the first step, the feedback word $s_{11}$ is computed from $s_{10}, s_{4}$ and $s_{1}$, and the internal state of the LFSR is updated, leading to a new state composed of $s_{2}$ to $s_{11}$.

- The first four output values are $z_{1}, z_{2}, z_{3}$ and $z_{4}$, and are computed using one application of Serpent1 over $\left(f_{4}, f_{3}, f_{2}, f_{1}\right)$, whose output is combined by XORs with $\left(s_{4}, s_{3}, s_{2}, s_{1}\right)$. 


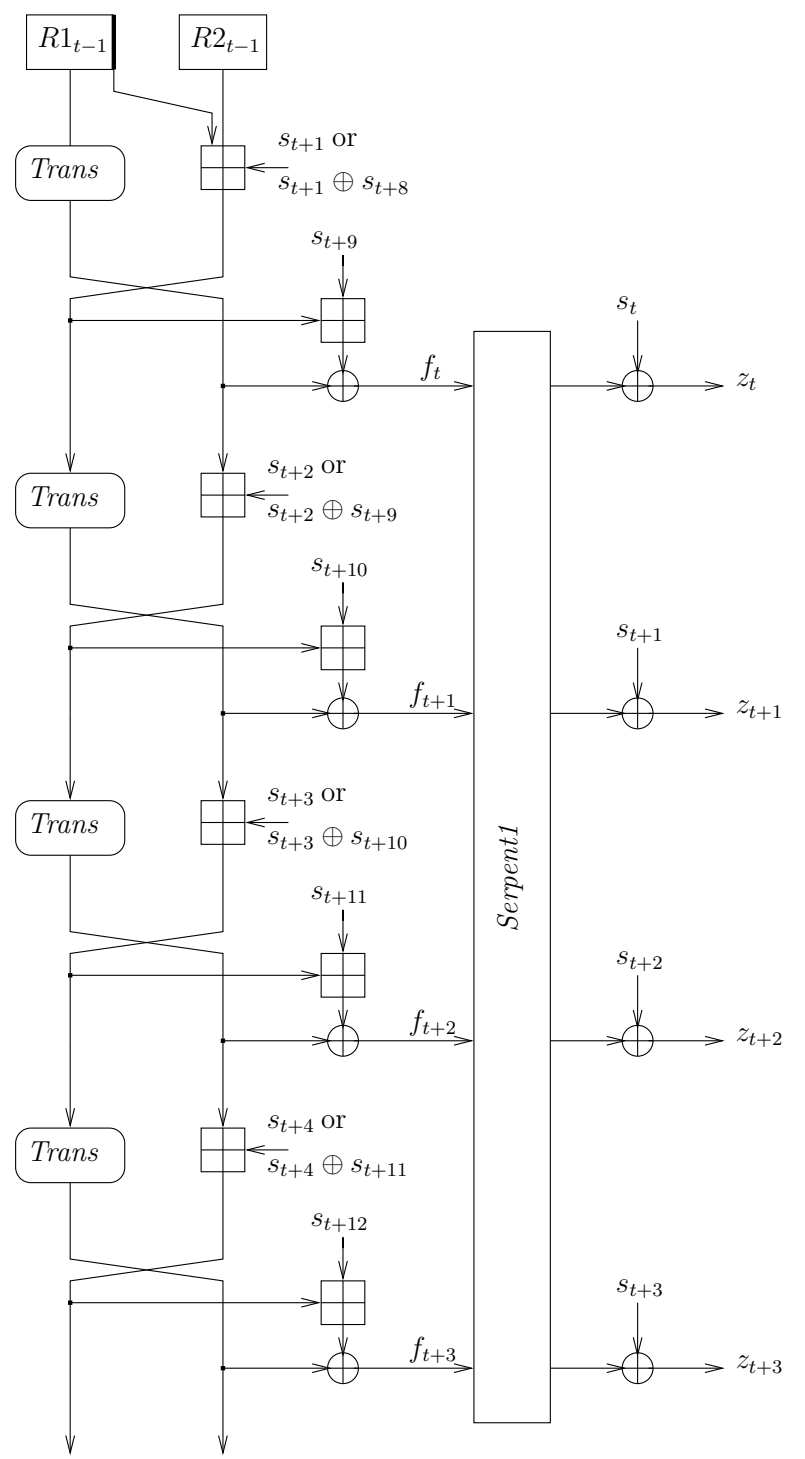

Fig. 2. The output transformation on four consecutive rounds of SoseMANUK. 


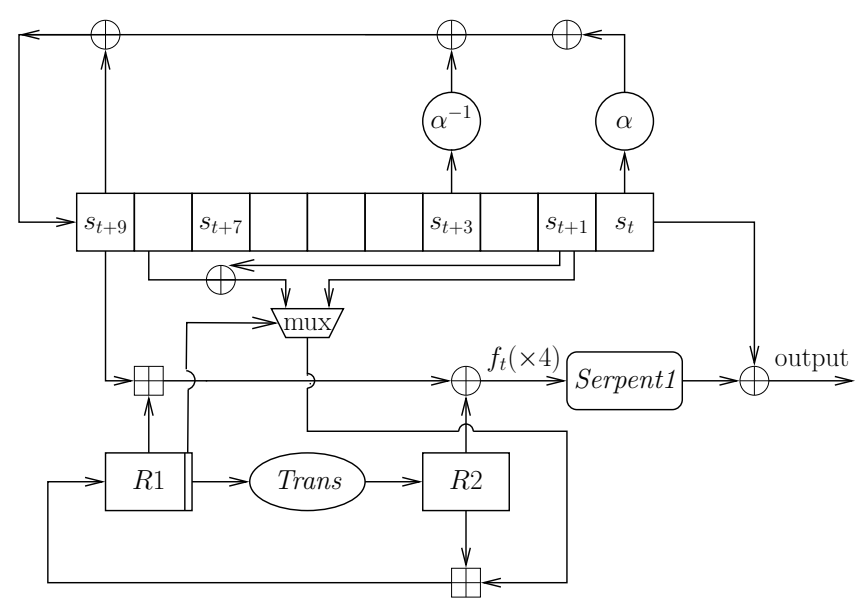

Fig. 3. An overview of Sosemanuk

\subsection{Key initialization and IV injection}

The Sosemanuk initialization process is split into two steps:

- the key schedule, which processes the secret key but does not depend on the IV; and

- the IV injection, which uses the output of the key schedule and the IV. This initializes the stream cipher internal state.

Key schedule The key setup corresponds to the Serpent24 key schedule, which produces 25 128-bit subkeys, as 100 32-bit words. These 25 128-bit subkeys are identical to the first 25128 -bit subkeys produced by the plain SERPENT key schedule.

SERPENT accepts any key length from 1 to 256 bits; hence, SosEMANUK may work with exactly the same keys. However, since SosEMANUK aims at 128-bit security; its key length must then be at least 128 bits. Therefore, 128 bits is the standard key length. Any key length from 128 bits to 256 bits is supported. But, the security level still corresponds to 128-bit security. In other words, using a longer secret key does not guarantee to provide the security level usually expected from such a key.

IV injection The IV is a 128-bit value. It is used as input to the Serpent24 block cipher, as initialized by the key schedule. Serpent24 consists 
of 24 rounds and the outputs of the 12th, 18th and 24th rounds are used. We denote those outputs as follows:

- $\left(Y_{3}^{12}, Y_{2}^{12}, Y_{1}^{12}, Y_{0}^{12}\right)$ : output of the 12 th round;

- $\left(Y_{3}^{18}, Y_{2}^{18}, Y_{1}^{18}, Y_{0}^{18}\right)$ : output of the 18th round;

- $\left(Y_{3}^{24}, Y_{2}^{24}, Y_{1}^{24}, Y_{0}^{24}\right)$ : output of the 24 th round.

The output of each round consists of the four 32-bit words just after the linear transformation, except for the 24th round, for which the output is taken just after the addition of the 25th subkey.

These values are used to initialize the SosEMANUK internal state, with the following values:

$$
\begin{aligned}
\left(s_{7}, s_{8}, s_{9}, s_{10}\right) & =\left(Y_{3}^{12}, Y_{2}^{12}, Y_{1}^{12}, Y_{0}^{12}\right) \\
\left(s_{5}, s_{6}\right) & =\left(Y_{1}^{18}, Y_{3}^{18}\right) \\
\left(s_{1}, s_{2}, s_{3}, s_{4}\right) & =\left(Y_{3}^{24}, Y_{2}^{24}, Y_{1}^{24}, Y_{0}^{24}\right) \\
R 1_{0} & =Y_{0}^{18} \\
R 2_{0} & =Y_{2}^{18}
\end{aligned}
$$

\section{Design rationale}

\subsection{Key initialization and IV injection}

Underlying principle. A first property of the initialization process is that it is split into two distinct steps: the key schedule which does not depend on the IV, and the IV injection which generates the initial state of the generator from the IV and from the output of the key schedule. Then, the IV setup for a fixed key is less expensive than a complete key setup, improving the common design since changing the IV is more frequent than changing the secret key.

A second characteristic of SosEmAnUK is that the IV setup is derived from the application of a block cipher over the IV. If we consider the function $F_{K}$ which maps a $n$-bit IV to the first $n$ bits of output stream generated from the key $K$ and the IV, then $F_{K}$ must be computationaly indistinguishable from a random function over $\mathbb{F}_{2}^{n}$. Hence, the computation of $F_{K}$ cannot "morally" be faster than the best known PRF over $n$-bit blocks. It so happens that the fastest known PRF use the same implementation techniques that the fastest known Pseudo-Random Permutations (which are block ciphers), and amount to the equivalent performance.

Since SosEMANUK stream generation is very fast, the generation of $n$ stream bits takes little time compared to a computation of a robust PRP over a block of $n$ bits. Following this path of reasoning, we decided to use 
a block cipher as the fundation of the IV setup for SosEmanuk: the IV setup itself cannot be much faster than the application of a block cipher, and the security requirements for that step are much similar to what is expected from a block cipher.

Choice of the block cipher. The block cipher used in the IV setup is derived from SERPENT for the following reasons:

- SERPENT has been thoroughly analyzed during the AES selection process and its security is well-understood.

- SERPENT needs no static data tables, and hence adds little or no data cache pressure.

- The SERPENT round function is optimized for operation over data represented as 32-bit words, which is exactly how data is managed within Sosemanuk. Using SERPENT implies no tedious byte extraction from 32-bit words, or recombinations into such words.

- We needed a block cipher for the key schedule and IV injection; using something other else than AES seems good for "biodiversity".

Design of Serpent24. The IV injection uses a reduced version of SERPENT because SERPENT aimed at 256-bit security, whereas SOSEMANUK is meant for 128-bit security. The best linear bias and differential bias for a 6 -round version of SERPENT are $2^{-28}$ and $2^{-58}$ respectively [3]. Thus, 12 rounds should provide appropriate security. Twelve more rounds are added in order to generate enough data (three 128-bit words are needed for initializing Sosemanuk), hence 24 rounds for Serpent24. We rely on the Sosemanuk core itself to provide some security margins (the output of Serpent24 is not available directly to the attacker). Two consecutive outputs of data are spaced with six inner rounds in order to prevent the existence of relations between the bits of the initial state and the secret key bits which could be used in an attack.

\subsection{LFSR}

The SNOW 2.0 LFSR contains 16 elements, which means 512 bits of internal state. Since we aim only at 128-bit security, we can accommodate a shorter LFSR. To defeat time-memory-data trade-off attacks, 256 bits of internal state at least should be used; we wanted some security margin, hence an LFSR length a bit more than six words. 
LFSR length. The LFSR length $n$ must be as small as possible: the bigger the state, the more difficult it is to map the state values on the processor registers. Ideally, the total state should fit in the 16 general-purpose registers that the new AMD64 architecture offers.

For efficient LFSR implementation, the LFSR must not be physically shifted; moving data around contributes nothing to actual security, and takes time. If $n$ is the LFSR length, then $k n$ steps (for some integer $k$ ) must be "unrolled", so that at each step only one LFSR cell is modified. Moreover, since Serpent1 operates over four successive output values, $k n$ corresponds to $\operatorname{lcm}(4, n)$ and it should be kept as small as possible, since a higher code size increases code cache pressure.

These considerations led us to $n=8$ or 10. But, an LFSR of length eight presents potential weaknesses which may be exploited in a guessand-determine attack (see Section 1.3). Therefore, a LFSR of length 10 is a suitable choice: the 384-bit internal state length should be enough; only 20 steps need to be unrolled for an efficient implementation. The total internal state fits in 12 registers, which should map fine on the new AMD64 architecture.

Feedback polynomial. The design criteria for the feedback polynomial are similar to those used in SNOW 2.0. Since the feedback polynomial must be as sparse as possible, we chose as in SNOW 2.0 a primitive polynomial of the form

$$
\pi(X)=c_{0} X^{10}+c_{a} X^{n-a}+c_{b} X^{n-b}+1,
$$

where $0<a<b<10$. The coefficients $c_{0}, c_{a}$ and $c_{b}$ preferably lie in $\left\{1, \alpha, \alpha^{-1}\right\}$ which are the elements corresponding to an efficient multiplication in $\mathbb{F}_{2^{32}}$. Moreover, $\left\{c_{0}, c_{a}, c_{b}\right\}$ must contain at least two distinct non-binary elements; otherwise, a multiple of $\pi$ with binary coefficients can be easily constructed 11, 16], providing an equation which holds for each single bit position.

We also want $a$ and $b$ to be coprime with the LFSR length. Otherwise, for instance if $d=\operatorname{gcd}(a, 10)>1$, the corresponding recurrence relation

$$
s_{t+10}=c_{b} s_{t+b}+c_{a} s_{t+a}+c_{0} s_{t}
$$

involves three terms of a decimated sequence $\left(s_{d t+i}\right)_{t>0}$ (for some integer $i$ ), which can be generated by an LFSR of length $n / d$ [23]. These conditions led us to $a=3$ and $b=9$. Since $a$ and $b$ are not coprime, $c_{a}$ and $c_{b}$ must be different; otherwise, some simplified relations may be exhibited by manipulating the feedback polynomial as shown in [16, 9]. The 
values $c_{0}=\alpha, c_{3}=\alpha^{-1}$ and $c_{9}=1$ correspond to a suitable primitive polynomial that fulfills all previously mentioned conditions.

\subsection{FSM}

The Trans function. The Trans function is chosen according to the following implementation criteria: no static data tables in order to reduce the cache pressure and the function must be fast on modern processors. For these reasons, the Trans function is composed of a 32-bit multiplication and a bitwise rotation which are both very fast. The 32-bit multiplication provides excellent "data mixing" compared to the number of clock cycles it consumes. The bitwise rotation avoids the existence of a linear relation between the least-significant bits of the inputs and the output of the FSM.

The operations involved in the Trans functions are incompatible with the other operations used in the FSM (addition over $\mathbb{Z}_{2^{32}}$, XOR operation). Actually, mixing operations on the ring and on the vector space disables associativity and commutativity laws. For instance,

$$
\begin{gathered}
\left(M \times\left(R 2_{t-1}+s_{t+1}\right.\right. \\
\left.\left.\quad \neq \quad \bmod 2^{32}\right) \bmod 2^{32}\right)_{<<<7} \\
\left(M \times\left(R 2_{t-1}\right) \bmod 2^{32}\right)_{<<<7}+\left(M \times\left(s_{t+1}\right) \bmod 2^{32}\right)_{<<<7} \bmod 2^{32} .
\end{gathered}
$$

The mux operation. The mux operation aims at increasing the complexity of fast correlation and algebraic attacks, since it decimates the FSM input sequence in an irregular fashion. Moreover, this operation can be implemented efficiently with either control bit extension and bitwise operations, or an architecture specific "conditional move" opcode. Modern $\mathrm{C}$ compilers know how to perform those optimizations when compiling the C conditional ternary operator "?:". This multiplexer is quite fast and requires no jump.

It is fitting that both LFSR elements $s_{t+c}$ and $s_{t+d}$ (with $c \leq d$ ) in the mux operation are not involved in the recurrence relation. Otherwise the complexity of guess-and-determine attacksmight be reduced. The distance $(d-c)$ between those elements must be coprime with the LFSR length since they must not be expressed as a decimated sequence with a lower linear complexity. Here, we choose $d-c=7$. Finally, it must be impossible for the inputs of the mux operation at two different steps correspond to the same element in the LFSR sequence. For this reason, the mux operation outputs either $s_{t+c}$ or $s_{t+c} \oplus s_{t+d}$. If $s_{t+c} \oplus s_{t+d}$ is the input of the FSM at time $t$, the possible inputs at time $(t+d-c)$ are $s_{t+d}$ and 
$s_{t+d} \oplus s_{t+2 d-c}$, which do not match any previous input. It is worth noticy that this property does not hold anymore if the mux outputs either $s_{t+c}$ or $s_{t+d}$.

\subsection{The output transformation}

The output transformation derived from Serpent1 aims at mixing four successive outputs of the FSM in a nonlinear way. As a consequence, any 32-bit keystream word produced by SosEmanuk depends on four consecutive intermediate values $f_{t}$. As a result, recovering any single output of the FSM, $f_{t}$, in a guess-and-determine attack requires the knowledge of at least four consecutive words from the LFSR sequence, $s_{t}, s_{t+1}, s_{t+2}, s_{t+3}$ (see Section 4.3 for details).

The following properties have also been taken into account in the choice of output transformation.

- Both nonlinear mixing operations involved in Sosemanuk (the Trans operation and the Serpent1 used in bitslice mode) do not provide any correlation probability or linear property on the least significant bits that could be used to mount an attack (see Section 4.4 for further details).

- From an algebraic point of view, those operations are combined to produce nonlinear equations (see Section 4.6).

- No linear relation can be directly exploited on the least significant bit of the values $\left(f_{t}, f_{t+1}, f_{t+2}, f_{t+3}\right)$, only quadratic equations with more variables than the number of possible equations (see Section 4.4).

- The linear relation between $s_{t}$ and $\operatorname{Serpent} 1\left(f_{t}, f_{t+1}, f_{t+2}, f_{t+3}\right)$ prevents SOSEMANUK from SQUARE-like attacks.

Finally, the fastest SERPENT S-box $\left(S_{2}\right)$ has been chosen in Serpent1 from an efficiency point of view [22]. But, $S_{2}$ also guarantees that there is no differential-linear relation on the least significant bit (the "most linear" one in the output of the FSM).

\section{Resistance against known attacks}

Our stream cipher SosEmanuK offers a 128-bit security, based on the following security model. 


\subsection{Security model}

The attacker is a probabilistic Turing Machine with access to a black box (oracle) that accepts the following three instructions: RESET, INIT with a 128-bit input, GetStream with a 1-bit output. The attacker's goal is to distinguish with probability $2 / 3$ between a black box that generates random output, and a black box that implements the stream cipher, where RESET generates a random key, INIT initializes the internal state of the stream cipher with a new chosen IV, and GetStream generates the next bit of keystream. The attacker is allowed to do $2^{128}$ elementary operations, an instruction to the black box being an elementary operation.

This security model falls under remarks made by Hong and Sarkar [18], because the precomputation time is not bounded by our model. Therefore our claim is that the 256-bit key variant of SosEmANuK provide a 128bit security. We do not know of a formal security model that restricts the precomputation time, i.e. that only allows the attacker one of the probabilistic Turing machines that can be built in a reasonable time from the current content of today's computers. Therefore, our claim is that the 128-bit key variant of SosEmAnuK, and all variants with larger keys, provide a 128-bit security against an attacker that is not allowed to benefit from large precomputation.

The following sections focus on the security of SosEmanuk against known attacks. It is important to note that the secret key of the cipher cannot be easily recovered from the initial state of the generator. Once the initial state is recovered, the attacker is only able to generate the output sequence for a particular key and a given IV. Recovering the secret key or generating the output for a different IV additionally requires the cost of an attack on Serpent24 with a certain number of plaintext/ciphertext pairs.

\subsection{Time-memory-data tradeoff attacks}

Due to the choice of the length of the LFSR (more than twice the key length), the time-memory-data tradeoff attacks described in [2,14,5] are impracticable. Moreover, since these TMDTO attacks aim at recovering the internal state of the cipher, recovering the secret key requires the additional cost of an attack against Serpent24. The best time-memory data tradeoff attack is the Hellman's one [17] which aims at recovering a pair $(K, I V)$. For a 128-bit secret key and a 128-bit IV, its time complexity is equal to $2^{128}$ cipher operations (see 18 for further details). 


\subsection{Guess and determine attacks}

The main weaknesses of SNOW 1.0 are related to this type of attacks (two at least have been exhibited [16], 9]). They essentially exploit a particular weakness in the linear recurrence equation. This does not hold anymore for the new polynomial choice in SNOW 2.0 and for the polynomial used in Sosemanuk which involve non-binary multiplications by two different constants. The first attack [16] also exploited a "trick" coming from the dependence between the values $R 1_{t-1}$ and $R 1_{t}$. This trick is avoided in SNOW 2.0 (because there is no direct link between those two register values anymore) and in Sosemanuk.

The best guess and determine attack we have found on Sosemanuk is the following.

- Guess at time $t, s_{t}, s_{t+1}, s_{t+2}, s_{t+3}, R 1_{t-1}$ and $R 2_{t-1}$ (6 words).

- Compute the corresponding outputs of the FSM $\left(f_{t}, f_{t+1}, f_{t+2}, f_{t+3}\right)$.

- Compute $R 2_{t}=\operatorname{Trans}\left(R 1_{t-1}\right)$ and $R 1_{t}$ from Equation (1) if $\operatorname{lsb}\left(R 1_{t-1}\right)=$ 1 (this can be done only with probability $1 / 2$ ).

- From $f_{t}=\left(s_{t+9}+R 1_{t} \bmod 2^{32}\right) \oplus R 2_{t}$, compute $s_{t+9}$.

- Compute $R 1_{t+1}$ from the knowledge of both $s_{t+2}$ and $s_{t+9}$; compute $R 2_{t+1}$. Compute $s_{t+10}$ from $f_{t+1}, R 1_{t+1}$ and $R 2_{t+1}$.

- Compute $R 1_{t+2}$ from $s_{t+3}$ and $s_{t+10}$; compute $R 2_{t+2}$. Compute $s_{t+11}$ from $f_{t+2}, R 1_{t+2}$ and $R 2_{t+2}$. Now, $s_{t+4}$ can be recovered due to the feedback relation at time $t+1$ :

$$
\alpha^{-1} s_{t+4}=s_{t+11} \oplus s_{t+10} \oplus \alpha s_{t+1} .
$$

- Compute $R 1_{t+3}$ from $s_{t+4}$ and $s_{t+11}$; compute $R 2_{t+2}$. Compute $s_{t+12}$ from $f_{t+3}, R 1_{t+3}$ and $R 2_{t+3}$. Compute $s_{t+5}$ by the feedback relation at time $t+2$ :

$$
\alpha^{-1} s_{t+5}=s_{t+12} \oplus s_{t+11} \oplus \alpha s_{t+2} .
$$

At this point, the LFSR words $s_{t}, s_{t+1}, s_{t+2}, s_{t+3}, s_{t+4}, s_{t+5}, s_{t+9}$ are known. Three elements $\left(s_{t+6}, s_{t+7}, s_{t+8}\right)$ remain unknown. To complete the full 10 words state of the LFSR, we need to guess 2 more words, $s_{t+6}$ and $s_{t+7}$ since each $f_{t+i}, 4 \leq i \leq 7$, depends on all 4 words $s_{t+4}, s_{t+5}$, $s_{t+6}$ and $s_{t+7}$. Therefore, this attack requires the guess of 832 -bit words, leading to a complexity of $2^{256}$.

Note that in [1] and in 25] the authors respectively proposed two guess and determine attacks against SOSEMANuK that have a complexity 
approximatively equal to $2^{226}$ and $2^{224}$ computations. However, as stated in paragraphs 2.6, 3.2 and 4.1, we never intended to have more than 128-bit security. The internal state of Sosemanuk is 384-bit long, which would be bad practice if we aimed at 256-bit security. Therefore, those guess-and-determine attacks, while being interesting theoretical studies, do not compromise the security of Sosemanuk.

\subsection{Correlation attacks}

In order to find a relevant correlation in SosEmanuk, the following questions can be addressed:

- does there exist a linear relation at bit level between some input and output bits?

- does there exist a particular relation between some input bit vector and some output bit vector?

In the first case, two linear relations could be exhibited at the bit level. In the first, the least significant bit of $s_{t+9}$ was "conserved", since the modular addition over $\mathbb{Z}_{232}$ is a linear operation on the least significant bit. The second linear relation induced by the FSM concerns the least significant bit of $s_{t+1}$ or of $s_{t+1} \oplus s_{t+8}$ (used to compute $R 1_{t}$ ) or the seventh bit of $R 2_{t}$ computed from $s_{t}$ or of $s_{t} \oplus s_{t+7}$. We here use that $R 2_{t}=\operatorname{Trans}\left(R 1_{t-1}\right)$ and $R 1_{t-1}=R 2_{t-2}+\left(s_{t}\right.$ or $\left.\left(s_{t} \oplus s_{t+7}\right)\right) \bmod 2^{32}$.

No linear relation holds after applying Serpent 1 and there are too many unknown bits to exploit a relation on the outputs words due to the bitslice design. Moreover, a fast correlation attack seems to be impracticable because the mux operation prevents certainty in the dependence between the LFSR states and the observed keystream.

\subsection{Distinguishing attacks}

A distinguishing attack by D. Coppersmith, S. Halevi and C. Jutla (see [10]) against the first version of SNOW used a particular weakness of the feedback polynomial built on a single multiplication by $\alpha$. This property does not hold for the choice of the new polynomial in SNOW 2.0 and for the polynomial used in Sosemanuk where multiplication by $\alpha^{-1}$ is also included.

In [26], D. Watanabe, A. Biryukov and C. De Cannière have mounted a new distinguishing attack on SNOW 2.0 with a complexity about $2^{225}$ 
operations using multiple linear masking method. They construct 3 different masks $\Gamma_{1}=\Gamma, \Gamma_{2}=\Gamma \cdot \alpha$ and $\Gamma_{3}=\Gamma \cdot \alpha^{-1}$ based on the same linear relation $\Gamma$.

The linear property deduced from the masks $\Gamma_{i}(i=1,2$ or 3$)$ must hold with a high probability on the both following quantities: $\Gamma_{i} \cdot S^{\prime}(x)=$ $\Gamma_{i} \cdot x$ and $\Gamma_{i} \cdot z \oplus \Gamma_{i} \cdot t=\Gamma_{i} \cdot(z \boxplus t)$ for $i=1,2$ and 3 , where $S^{\prime}$ is the transition function of the FSM in SNOW 2.0. In the case of SNOW 2.0, the hardest hypothesis to satisfy is the first one defined on $y=S^{\prime}(x)$. In the case of Sosemanuk, we need $\operatorname{Pr}\left(\Gamma_{i} \cdot \operatorname{Trans}(x)=\Gamma_{i} \cdot x\right)_{i=1,2,3}$ to be high. But, we also need that $\forall i=1,2,3$, the relation

$$
\left(\Gamma_{i}^{\prime}, \Gamma_{i}^{\prime}, \Gamma_{i}^{\prime}, \Gamma_{i}^{\prime}\right) \cdot\left(x_{1}, x_{2}, x_{3}, x_{4}\right)=\operatorname{Serpent1}\left(\left(\Gamma_{i}, \Gamma_{i}, \Gamma_{i}, \Gamma_{i}\right) \cdot\left(x_{1}, x_{2}, x_{3}, x_{4}\right)\right) .
$$

for some $\Gamma_{i}^{\prime} \in \mathbb{F}_{2}^{32}$, holds with a high probability.

Due to the bitslice design chosen for Serpent1, it seems very difficult to find such a mask. Therefore, the attack described in [26] could not be applied directly on Sosemanuk.

\subsection{Algebraic attacks}

Let us consider, as in 画, the initial state of the LFSR at bit level:

$$
\left(s_{10}, \cdots, s_{1}\right)=\left(s_{10}^{31}, \cdots, s_{10}^{0}, \cdots, s_{1}^{31}, \cdots, s_{1}^{0}\right)
$$

Then, the outputs of SosEmanuk at time $t \geq 1$ could be written:

$$
F^{t}\left(\left(s_{31}^{10}, \cdots, s_{0}^{1}\right)\right)=\left(z_{t}, z_{t+1}, z_{t+2}, z_{t+3}\right)
$$

where $F$ is a vectorial Boolean function from $\mathbb{F}_{2}^{320}$ into $\mathbb{F}_{2}^{128}$ that could be seen as 128 Boolean functions $F_{j}, \forall j \in[0 . .127]$ from $\mathbb{F}_{2}^{320}$ into $\mathbb{F}_{2}$.

Let us study the degree of an $F_{j}$ function depending on a particular bit of the output or on a linear combination of output bits because it is not possible to directly compute the algebraic immunity of each function $F_{j}$ due to the very large number of variables (320 input bits). We think that the following remarks prevent the existence of low degree relations between the inputs and the outputs of $F_{j}$.

- The output bit $i$ after the modular addition on $\mathbb{Z}_{2^{32}}$ is of degree $i+1$ (as described in [6]).

- The output bit $i$ after the Trans mapping is of degree $i+1-7 \bmod 32, \forall i \neq$ 6 and equal to 32 for $i=6$ (as described in [6]). 
- The mux operation does not enable to determine with probability one the exact number of bits of the initial state involved in the algebraic relation.

- The algebraic immunity of the SERPENT S-box $S_{2}$ at 4-bit word level is equal to 2 (see 21] for a definition of the algebraic immunity and more details).

Under those remarks, we think that an algebraic attack against SosEMANUK is intractable.

\section{Implementation}

The reference $\mathrm{C}$ implementation is also an optimized implementation. When compiled with the SOSEMANUK_VECTOR macro defined, it is a full program (with its own main() function) which outputs two detailed test vectors. Since the LFSR length is ten, we unroll the $\mathrm{C}$ code on 20 rounds (see 3.2 for details); each test vector contains:

- A copy of the secret key (a sequence of bytes, expressed in hexadecimal).

- The expanded secret key, as described by the SERPENT specification: the key is expanded to 256 bits, then read as a 256-bit number with the little endian convention. The test vector outputs that key as a big hexadecimal number, with some digit grouping.

- The 25 Serpent24 subkeys, each of them consisting of four 32-bit words (in the $\left(K_{3}, K_{2}, K_{1}, K_{0}\right)$ order).

- The 128-bit IV, as a sequence of 16 bytes.

- The IV, once transformed into four 32-bit words, in the $\left(I_{3}, I_{2}, I_{1}, I_{0}\right)$ order.

- The initial LFSR state ( $s_{1}$ to $s_{10}$, in that order).

- The initial FSM state $\left(R 1_{0}\right.$ and $\left.R 2_{0}\right)$.

- Ten times the following data:

- Four times the following:

* the new FSM state $\left(R 1_{t}\right.$ and $\left.R 2_{t}\right)$;

* the new LFSR state, after the update (the dropped value $s_{t}$ is also output);

* the intermediate output $f_{t}$.

- The Serpent1 input.

- The Serpent1 output.

- 16 bytes of Sosemanuk output.

- The total stream output (160 bytes). 


\section{Performance}

\subsection{Software implementation}

This section is devoted to the software performance of Sosemanuk. It compares the performance of SOSEMANUK with the other candidates selected in the Phase 3 (Software Profile), SNOW 2.0 and AES-CTR using the eSTREAM testing framework and the provided reference $\mathrm{C}$ implementations [7]. The three tables Table 1, Table 2 and Table 3 sum up the results (for the keystream generation, the $I V$ setup and the key setup) given in [8] for three different architectures: an Intel Pentium 4 (CISC target), an AMD Athlon64 X2 4200+ (CISC target) and an Alpha EV6 (RISC target).

All the results presented for Sosemanuk have been computed using the supplied reference $\mathrm{C}$ implementation.

Code size. The main unrolled loop implies a code size between 2 and 5 $\mathrm{KB}$ depending on the platform and the compiler. Therefore, the entire code fits in the L1 cache.

Static data. The reference C implementation uses static data tables with a total size equal to $4 \mathrm{~KB}$. This amount is 3 times smaller than the size of static data required in SNOW 2.0, leading to a lower date cache pressure.

Key setup. We recall that the key setup (the subkey generation given by Serpent24) is made once and that each new IV injection for a given key corresponds to a small version of the block cipher SERPENT.

The performance of the key setup and of the IV setup in SosemanuK are directly derived from the performance of SERPENT [13]. Due to intellectual property aspects, our reference implementation does not reuse the best implementation of SERPENT. However, the performance given in 20] (i.e., computed on the Gladman's code written in assembly language [13]) leads to the following results on a Pentium 4:

- key setup $\simeq 900$ cycles;

- IV setup $\simeq 480$ cycles.

These estimations for the IV setup (resp. key setup) performance corresponds to about $3 / 4$ of the best published performance for SERPENT encryption (resp. for SERPENT key schedule). 
Performance results. Table 1, Table 2 and Table 3 present the performance of the keystream generation (using four performance measures), the agility, the $I V$ setup and the key setup to test the most relevant implementation properties. The four elementary tests for keystream generation are: the encryption rate for long streams by ciphering a long stream in chunks of about $4 \mathrm{~Kb}$; the packet encryption rate for three packet lengths (40, 576 and 1500 bytes) including an $I V$ setup; the agility test initiates a large number of sessions (filling 16MB of RAM), and then encrypts streams of plaintexts in short blocks of around 256 bytes, each time jumping from one session to another.

\begin{tabular}{|c|c|c|c|c|c|c|c|c|c|}
\hline & & & \multicolumn{5}{|c|}{ cycles/byte } & cycles/key & cycles/IV \\
\hline Algo. & Key & $I V$ & Stream & 40 bytes & 576 bytes & 1500 bytes & agility & Key setup & IV setup \\
\hline AES CTR & 128 & 128 & 17.81 & 29.19 & 18.35 & 18.04 & 20.77 & 393.45 & 76.16 \\
\hline SNOW v2.0 & 128 & 128 & 5.04 & 35.60 & 6.92 & 5.92 & 7.95 & 85.44 & 1000.54 \\
\hline CryptMT (v3) & 128 & 128 & 5.27 & 39.12 & 12.09 & 11.55 & 11.35 & 53.71 & 849.25 \\
\hline DRAGON & 128 & 128 & 11.37 & 74.09 & 26.07 & 23.23 & 15.00 & 256.04 & 1925.54 \\
\hline HC-128 & 128 & 128 & 3.76 & 1458.58 & 104.86 & 42.64 & 19.02 & 78.81 & 56929.45 \\
\hline HC-256 & 128 & 128 & 4.39 & 2596.20 & 184.25 & 73.59 & 26.27 & 76.66 & 104341.33 \\
\hline LEXv1 & 128 & 128 & 9.46 & 20.78 & 10.88 & 10.01 & 12.30 & 486.57 & 449.00 \\
\hline NLSv2 & 128 & 128 & 6.64 & 38.94 & 8.52 & 6.97 & 12.10 & 823.74 & 704.68 \\
\hline Rabbit & 128 & 64 & 9.46 & 34.45 & 11.77 & 10.76 & 12.89 & 984.27 & 825.55 \\
\hline Salsa20 & 128 & 64 & 16.61 & 42.21 & 17.63 & 18.57 & 18.71 & 90.32 & 78.19 \\
\hline SOSEMANUK & 128 & 64 & 5.81 & 52.37 & 12.52 & 9.62 & 7.40 & 1287.55 & 1245.71 \\
\hline
\end{tabular}

Table 1. Number of CPU cycles for the stream ciphers using a Pentium 4 at $2.80 \mathrm{GHz}$, Model 15/2/9

As shown in these tables, Sosemanuk remains among the fastest algorithms on several platforms due to a good design for the mappings of data on the processor registers and a low data cache pressure.

\subsection{Hardware implementation}

In [15], the authors propose hardware implementations and performance metrics for several stream cipher candidates and especially SosEmANUK. They remark that even if the design of SosEmanuK is a little bit complex to implement, it leads to an impressive performance. The required number of gates for designing SosemanuK on $0.13 \mu \mathrm{m}$ Standard Cell CMOS with a key of length 256 bits is 18819 considering that 32 bits are outputted at each cycle. Moreover, the corresponding leakage power is $33.55 \mu \mathrm{W}$ for a 


\begin{tabular}{|c|c|c|c|c|c|c|c|c|c|}
\hline & & & \multicolumn{5}{|c|}{ cycles/byte } & cycles/key & cycles/IV \\
\hline Algo. & Key & $I V$ & Stream & 40 bytes & 576 bytes & 1500 bytes & agility & Key setup & IV setup \\
\hline AES CTR & 128 & 128 & 13.39 & 18.09 & 13.39 & 13.35 & 15.03 & 152.81 & 15.58 \\
\hline SNOW v2.0 & 128 & 128 & 4.83 & 23.18 & 5.77 & 5.34 & 6.46 & 43.37 & 528.04 \\
\hline CryptMT (v3) & 128 & 128 & 4.65 & 19.26 & 8.47 & 7.64 & 8.82 & 25.47 & 384.33 \\
\hline DRAGON & 128 & 128 & 7.76 & 60.20 & 25.90 & 24.31 & 10.01 & 89.90 & 1449.74 \\
\hline HC-128 & 128 & 128 & 2.86 & 587.00 & 43.19 & 18.43 & 13.07 & 37.85 & 23308.78 \\
\hline HC-256 & 128 & 128 & 4.72 & 1420.99 & 103.10 & 42.83 & 21.13 & 41.31 & 56725.89 \\
\hline LEXv1 & 128 & 128 & 6.84 & 14.19 & 7.78 & 7.20 & 9.19 & 226.41 & 268.31 \\
\hline NLSv2 & 128 & 128 & 10.69 & 53.24 & 13.45 & 11.48 & 14.13 & 453.35 & 1293.15 \\
\hline Rabbit & 128 & 64 & 4.98 & 14.60 & 5.55 & 5.25 & 6.34 & 288.21 & 292.38 \\
\hline Salsa20 & 128 & 64 & 7.64 & 16.10 & 7.74 & 7.91 & 8.93 & 24.57 & 14.29 \\
\hline SOSEMANUK & 128 & 64 & 4.07 & 25.26 & 7.20 & 6.10 & 5.12 & 759.06 & 560.63 \\
\hline
\end{tabular}

Table 2. Number of CPU cycles for the stream ciphers using an AMD Athlon 64 X2 $4200+$ at 2.20GHz, Model 15/75/2

\begin{tabular}{|c|c|c|c|c|c|c|c|c|c|}
\hline & & & \multicolumn{5}{|c|}{ cycles/byte } & cycles/key & cycles/IV \\
\hline Algo. & Key & $I V$ & Stream & 40 bytes & 576 bytes & 1500 bytes & agility & Key setup & IV setup \\
\hline AES CTR & 128 & 128 & 15.53 & 24.63 & 15.94 & 15.82 & 17.80 & 633.65 & 37.58 \\
\hline SNOW v2.0 & 128 & 128 & 5.17 & 23.74 & 6.11 & 5.73 & 6.37 & 69.00 & 489.35 \\
\hline CryptMT (v3) & 128 & 128 & 6.90 & 24.74 & 11.64 & 11.75 & 12.86 & 37.49 & 422.17 \\
\hline DRAGON & 128 & 128 & 8.46 & 74.94 & 41.89 & 40.52 & 10.13 & 234.33 & 1542.46 \\
\hline HC-128 & 128 & 128 & 3.90 & 1029.93 & 77.41 & 31.59 & 14.80 & 54.67 & 42130.00 \\
\hline HC-256 & 128 & 128 & 5.18 & 2414.77 & 171.48 & 69.34 & 23.53 & 52.96 & 95937.00 \\
\hline LEXv1 & 128 & 128 & 7.99 & 16.87 & 9.15 & 8.44 & 9.53 & 198.49 & 334.58 \\
\hline NLSv2 & 128 & 128 & 5.93 & 24.26 & 6.44 & 5.59 & 7.94 & 530.39 & 421.66 \\
\hline Rabbit & 128 & 64 & 5.27 & 14.49 & 5.69 & 5.53 & 6.32 & 318.57 & 280.63 \\
\hline Salsa20 & 128 & 64 & 13.61 & 39.93 & 13.77 & 14.34 & 14.46 & 33.60 & 20.16 \\
\hline SOSEMANUK & 128 & \begin{tabular}{|l|}
64 \\
\end{tabular} & 4.63 & 28.80 & 7.66 & 6.26 & 5.32 & 1301.09 & 692.71 \\
\hline
\end{tabular}

Table 3. Number of CPU cycles for the stream ciphers using an Alpha EV6 at $500 \mathrm{MHz}$, Model 21264

total power at $10 \mathrm{MHz}$ equal to $812.47 \mu \mathrm{W}$. The authors also derive the metrics for maximum clock frequency and for an output rate at $10 \mathrm{Mbps}$ (estimated typical future wireless LAN). In this last case, the corresponding clock frequency is equal to $0.313 \mathrm{MHz}$ for a Power-Area-Time equal to 564.8 nJ-um2. In conclusion, they recommend SosEMANUK for WLAN applications with a key length equal to 256 bits. They say that "with regard to Sosemanuk, the utility as a hardware cipher is clear thus in our opinion requires adding to the hardware focus profile." 


\section{Strengths and advantages of SOSEMANUK}

The new synchronous stream cipher SosEmanuk based upon the SNOW 2.0 design improves it from several points of view. From a security point of view, Sosemanuk avoids some potential weaknesses as the distinguishing attack proposed in [26] due to the particular use of Serpent1 in bitslice mode. The chosen LFSR is designed to eliminate all potential weaknesses (particular decimation properties, linear relations,...). The mappings used in the Finite State Machine have been carefully designed in the following way:

- The Trans function guarantees good properties of confusion and diffusion for a low cost in software. Moreover, this mapping prevents SosemanuK from algebraic attacks.

- The mux operation, that could be efficiently implemented, protects SosEmANuK from fast correlation attacks and algebraic attacks.

The Serpent1 output transformation, very efficient in bitslice mode, provides nonlinear equations, a good diffusion and it improves the resistance to guess-and-determine attacks.

The new design chosen for the key setup and the IV injection allows to split the initialization procedure into two distinct parts, without any loss of security. It leads to a much faster resynchronization mechanism.

From an efficiency point of view, due to a reduced amount of static data and a reduced internal state size, the exploitation of the processor registers is enhanced and the data cache pressure is improved on several platforms, especially on RISC architectures.

Acknowledgments The authors would like to thank Matt Robshaw for valuable comments.

Note that this work was done while the 4th author was affiliated to Axalto/Gemalto (France), the 7 th and the 12th authors were affiliated to France Télécom R\&D/Orange Labs (France), the 8th author was affiliated to the École Normale Supérieure (France), the 10th author was affiliated to INRIA Rocquencourt (France).

\section{References}

1. Hadi Ahmadi, Taraneh Eghlidos, and Shahram Khazaei. Improved guess and determine attack on SOSEMANUK. eSTREAM, ECRYPT Stream Cipher Project, Report 2005/085, 2005. http://www.ecrypt.eu.org/stream. 
2. S. Babbage. A space/time trade-off in exhaustive search attacks on stream ciphers. In European Convention on Security and Detection, number 408. IEEE Conference Publication, 1995.

3. E. Biham, R. Anderson, and L. Knudsen. SERPENT: A new block cipher proposal. In Fast Software Encryption - FSE'98, volume 1372 of Lecture Notes in Computer Science, pages 222-238. Springer-Verlag, 1998.

4. O. Billet and H. Gilbert. Resistance of SNOW 2.0 against algebraic attacks. In Topics in Cryptology - CT-RSA 2005, volume 3376 of Lecture Notes in Computer Science, pages 19-28. Springer-Verlag, 2005.

5. A. Biryukov and A. Shamir. Cryptanalytic time-memory-data trade-offs for stream ciphers. In Advances in Cryptology - ASIACRYPT 2000, volume 1976 of Lecture Notes in Computer Science, pages 1-14. Springer-Verlag, 2000.

6. A. Braeken and I. Semaev. The ANF of the composition of $\times$ and $+\bmod 2^{n}$ with a Boolean function. In Fast Software Encryption - FSE 2005, Lecture Notes in Computer Science. Springer-Verlag, 2005. To appear.

7. C. De Cannière. estream optimized code HOWTO. eSTREAM, ECRYPT Stream Cipher Project, 2005. http://www.ecrypt.eu.org/stream/perf/.

8. C. De Cannière. Software performance of the phase 3 candidates. eSTREAM, ECRYPT Stream Cipher Project, 2007. http://www.ecrypt.eu.org/stream/ phase3perf.html.

9. C. De Cannière. Guess and determine attack on SNOW - NESSIE public reports. https://www.cosic.esat.kuleuven.ac.be/nessie/reports/, 2001.

10. D. Coppersmith, S. Halevi, and C. Jutla. Cryptanalysis of stream ciphers with linear masking. In Advances in Cryptology - CRYPTO 2002, volume 2442 of Lecture Notes in Computer Science. Springer-Verlag, 2002.

11. P. Ekdahl and T. Johannson. Distinguishing attacks on SOBER. In Fast Software Encryption - FSE 2002, volume 2365 of Lecture Notes in Computer Science, pages 210-224. Springer-Verlag, 2002.

12. P. Ekdahl and T. Johansson. A new version of the stream cipher SNOW. In Selected Areas in Cryptography - SAC 2002, volume 2295 of Lecture Notes in Computer Science, pages 47-61. Springer-Verlag, 2002.

13. B. Gladman. SERPENT performance. http://fp.gladman.plus.com cryptography_technology/serpent/.

14. J. Golić. Cryptanalysis of alleged A5 stream cipher. In Advances in Cryptology - EUROCRYPT'97, volume 1233 of Lecture Notes in Computer Science, pages 239-255. Springer-Verlag, 1997.

15. T. Good and M. Benaissa. Hardware results for selected stream cipher candidates. eSTREAM, ECRYPT Stream Cipher Project, SASC 2007, Report 2007/023, 2007. http://www.ecrypt.eu.org/stream.

16. P. Hawkes and G. Rose. Guess-and-determine attacks on SNOW. In Selected Areas in Cryptography - SAC 2002, volume 2595 of Lecture Notes in Computer Science, pages 37-46. Springer-Verlag, 2002.

17. M. E. Hellman. A cryptanalytic time-memory trade-off. IEEE Transactions on Information Theory, 26(4):401-406, 1980.

18. J. Hong and P. Sarkar. Rediscovery of time memory tradeoffs. http://eprint. iacr.org/2005/090.ps, 2005.

19. K. Howard. Snow snake demonstration gives history lesson. http://www. turtletrack.org/Issues01/Co02102001/C0_02102001_Snowsnake.htm.

20. M. Matsui and S. Fukuda. How to maximize software performance of symmetric primitives on Pentiums. In Fast Software Encryption - FSE 2005, Lecture Notes in Computer Science. Springer-Verlag, 2005. to appear. 
21. W. Meier, E. Pasalic, and C. Carlet. Algebraic attacks and decomposition of Boolean functions. In Advances in Cryptology - EUROCRYPT 2004, volume 3027 of Lecture Notes in Computer Science, pages 474-491. Springer-Verlag, 2004.

22. D. Osvik. Speeding up SERPENT. http://www.ii.uib.no/ osvik/, April 2000. Second AES Candidate Conference.

23. R.A. Rueppel. Analysis and Design of stream ciphers. Springer-Verlag, 1986.

24. The story of Snowsnake. http://www.members.shaw.ca/dmacauley/story_of_ snowsnake.htm.

25. Yukiyasu Tsunoo, Teruo Saito, Maki Shigeri, Tomoyasu Suzaki, Hadi Ahmadi, Taraneh Eghlidos, and Shahram Khazaei. Evaluation of SOSEMANUK with regard to guess-and-determine attacks. eSTREAM, ECRYPT Stream Cipher Project, Report 2006/009, 2005. http://www.ecrypt.eu.org/stream.

26. D. Watanabe, A. Biryukov, and C. De Cannière. A distinguishing attack of SNOW 2.0 with linear masking method. In Selected Areas in Cryptography 2003, volume 3006 of Lecture Notes in Computer Science, pages 222-233. Springer-Verlag, 2003.

\section{A Specifications of SERPENT}

In this appendix, a recall on the specifications of SERPENT given in [3] is made. First, the S-boxes definition is given and the linear part is also defined again.

\section{A.1 S-boxes definitions}

The eight SERPENT S-boxes act on 4-bit words and are defined as permutations of $\mathbb{Z}_{16}$ :

$S 0: 3,8,15,1,10,6,5,11,14,13,4,2,7,0,9,12$

$S 1: 15,12,2,7,9,0,5,10,1,11,14,8,6,13,3,4$

$S 2: 8,6,7,9,3,12,10,15,13,1,14,4,0,11,5,2$

$S 3: 0,15,11,8,12,9,6,3,13,1,2,4,10,7,5,14$

$S 4: 1,15,8,3,12,0,11,6,2,5,4,10,9,14,7,13$

$S 5: 15,5,2,11,4,10,9,12,0,3,14,8,13,6,7,1$

$S 6: 7,2,12,5,8,4,6,11,14,9,1,15,13,3,10,0$

$S 7: 1,13,15,0,14,8,2,11,7,4,12,10,9,3,5,6$

\section{A.2 Linear part of SERPENT round function}

The linear part of a one round version of SERPENT acts on 4 32-bit words $\left(X_{3}, X_{2}, X_{1}, X_{0}\right)$ where $X_{0}$ is the least significant word and is defined as follows:

$$
\begin{aligned}
& X_{0}=X_{0}<<<13 \\
& X_{2}=X_{2}<<<3
\end{aligned}
$$




$$
\begin{aligned}
& X_{1}=X_{1} \oplus X_{0} \oplus X_{2} \\
& X_{3}=X_{3} \oplus X_{2} \oplus\left(X_{0}<<<3\right) \\
& X_{1}=X_{1}<<<1 \\
& X_{3}=X_{3}<<<7 \\
& X_{0}=X_{0} \oplus X_{1} \oplus X_{3} \\
& X_{2}=X_{2} \oplus X_{3} \oplus\left(X_{1}<<<7\right) \\
& X_{0}=X_{0}<<<5 \\
& X_{2}=X_{2}<<<22
\end{aligned}
$$

